\title{
Effectiveness of Two Types of Photostimulable Phosphor Plate Plastic Barrier Envelopes for Prevention of Microbiological Contamination
}

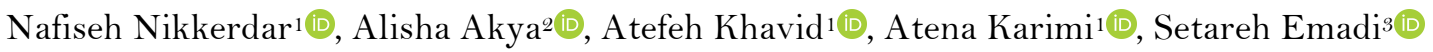

\begin{abstract}
${ }^{1}$ Department of Oral and Maxillofacial Radiology, School of Dentistry, Kermanshah University of Medical Sciences, Kermanshah, Iran.

${ }^{2}$ Nosocomial Infections Research Center, School of Medicine, Kermanshah University of Medical Sciences, Kermanshah, Iran.

${ }^{3}$ School of Dentistry, Kermanshah University of Medical Sciences, Kermanshah, Iran.
\end{abstract}

Author to whom correspondence should be addressed: Atena Karimi, Department of Oral and Maxillofacial Radiology, School of Dentistry, Kermanshah University of Medical Sciences, Kermanshah, IR Iran. Phone: +098 9127794870. E-mail: atenakarimi83@yahoo.com.

Academic Editors: Alessandro Leite Cavalcanti and Wilton Wilney Nascimento Padilha

Received: 06 November 2019 / Accepted: 29 February 2020 / Published: 12 March 2020

How to cite this article: Nikkerdar N, Akya A, Khavid A, Karimi A, Emadi S. Effectiveness of two types of photostimulable phosphor plate plastic barrier envelopes for prevention of microbiological contamination. Pesqui Bras Odontopediatria Clín Integr. 2020; 20:e5378. https://doi.org/10.1590/pboci.2020.047

\begin{abstract}
Objective: To compare the effectiveness of two types of commercially available photostimulable phosphor plate (PSP) protective barrier envelopes to prevent microbiological contamination. Material and Methods: In this cross-sectional study, 80 barrier envelopes were tested in 40 volunteers. The PSP plates were placed individually in Asia Teb and Soredex protective barrier envelopes and were placed in the mouth for two minutes, similar to periapical films. The protective barrier envelopes were then removed under sterile conditions, and the sensors were placed on different culture media. The number of colonies on each plate was counted. Data were analyzed using SPSS via McNemar and Wilcoxon tests. Results: Bacterial growth was noted in $17.5 \%$ of PSPs with Soredex, and $32.5 \%$ of PSPs with Asia Teb barrier envelopes. Grampositive bacilli were the most commonly isolated bacteria. The difference between the Asia Teb and Soredex barrier envelopes for the protection of microbiological contamination was not significant ( $\mathrm{p}>0.05)$. Conclusion: The use of different types of protective barrier envelopes was not sufficient for prevention of microbiological contamination of PSP plates, and some adjunct modalities were required to decrease microbiological contamination of PSP plates.
\end{abstract}

Keywords: Microbiology; Radiography, Dental, Digital; Gram-Positive Bacteria. 


\section{Introduction}

Intraoral radiography plays an important role in detection of carious lesions, periodontal disease and other pathological lesions [1]. In the past, conventional film-based radiography was the most commonly used dental imaging modality. Digital sensors were introduced in the late 1980s [2,3], and have included solid-state sensors namely charge-coupled devices (CCDs), complementary metal oxide semiconductor (CMOS), and photostimulable phosphor (PSP) plates [4].

The advantages of digital imaging systems have included the elimination of chemical processing, fast image retrieval, higher image quality, decreased environmental contamination and lower patient radiation dose compared to conventional radiography [5,6]. Although radiography is a non-invasive modality, film and sensors (PSP plates, CCDs and CMOS) can be easily contaminated with blood and saliva during radiography, and cause cross-contamination [7-12]. In a dental office setting, patients and the staff are at risk of infections with HIV, hepatitis B and C, Mycobacterium tuberculosis, Staphylococcus aureus, Streptococcus pneumonia and Treponema pallidum [13].

Digital receptors are reusable, but they cannot be sterilized [2,14-16]. This may cause crosscontamination in the clinical setting. Some measures have been taken to decrease the risk of infection transmission such as wearing protective gloves for placing the film/sensor in the oral cavity, and when removing the barrier envelope, disinfection of surfaces or use of protective barrier envelopes and sterilization of film holders [17,18].

Superficial disinfection of digital sensors has also been emphasized [19]. Currently, a commonly practiced infection control measure in digital radiography is the use of sealable plastic barrier envelopes, which are highly popular [20]. These barrier envelopes are available for PSP plates, CCDs and CMOS, and prevent infection transmission [9]. However, plastic barrier envelopes alone may not suffice for prevention of contamination of PSP plates by the oral microorganisms [10,14]. Contamination may occur in case of perforation of the plastic barrier envelope or contact of receptor with hand or blood when removing the barrier envelope.

Therefore, considering the availability of different types of barrier envelopes for digital receptors in the market and their wide price range, it is imperative to assess their effectiveness. This study aimed to compare the effectiveness of two types of protective barrier envelopes for PSP plates for prevention of microbiological contamination.

\section{Material and Methods}

\section{Study Design and Sample}

This analytical cross-sectional study evaluated microbiological contamination of PSP plates following the use of two types of protective barrier envelopes manufactured by PSP Optime (Soredex Imaging Plate, Helsinki, Finland) and (Asia Teb, Tehran, Iran). A total of 40 volunteers were selected among those presenting to the Radiology Department of Kermanshah University, School of Dentistry.

\section{Data Collection}

Previously used plates were autoclave-sterilized and used in this study. Two plastic barrier envelopes by the aforementioned two manufacturers were used for each candidate. The PSP plates along with the barrier envelopes were placed in the oral cavity at the site of the mandibular second premolar for two minutes to simulate taking a periapical radiograph with the bisecting angle technique, but no exposure was performed. 
After removing from the oral cavity, the plates were taken out of the barrier envelopes under sterile conditions (using sterile gloves and forceps), and placed in a sterile container.

\section{Microbiology Analysis}

The barrier envelopes were transferred to a microbiological laboratory in an icebox. In the laboratory, the PSP plates were placed in a sterile container containing $3 \mathrm{~mL}$ of tryptic soy broth (Merck KGaA, Darmstadt, Germany) such that the plates were dipped in the liquid culture medium. Three dilutions of 1/10, $1 / 100$ and 1/000 were prepared of the culture medium, and $200 \mu \mathrm{L}$ of each dilution was cultured on tryptic soy agar, MacConkey agar and mannitol salt agar (Merck KGaA, Darmstadt, Germany), and incubated for 48 hours. The number of colonies was then counted using a colony counter [21]. The final count of bacterial colonies was determined taking into account the dilution coefficient of each sample. Also, all colonies were identified by Gram staining.

\section{Data Analysis}

Data were entered into Excel software and analyzed using SPSS version 18 (SPSS Inc., IL, USA). The level of significance was considered at 0.05. Descriptive statistics, McNemar's test and Wilcoxon test were used.

Ethical Clearance

All participants signed informed consent forms. The approval protocol number was IR.KUMS.REC.1395.369.

\section{Results}

Of 40 participants, $20(50 \%)$ were males, and $20(50 \%)$ were females. The mean age of participants was $33.49 \pm 12.64$ years (range 16 to 66 years).

Bacterial growth was noted in 13 samples (32.5\%) in Asia Teb and seven samples (17.5\%) in Soredex group. Also, $60 \%$ of the Asia Teb and Soredex barrier envelopes did not show bacterial contamination. However, bacterial contamination was noted in $10 \%$ of the samples in both groups. Contamination with Grampositive bacilli had the highest frequency (Table 1).

The level of contamination did not have a normal distribution in Asia Teb or Soredex group $(\mathrm{p}<0.001)$. McNemar's test found no significant difference in bacterial growth between the two groups of Asia Teb and Soredex $(\mathrm{p}=0.146)$. Wilcoxon test showed that the level of contamination of Soredex and Asia Teb barrier envelopes was not significantly different $(p=0.689)$.

Table 1. Frequency of bacterial growth and proliferation in plastic barrier envelopes.

\begin{tabular}{lcc}
\hline \multicolumn{1}{c}{ Type of Colony } & Soredex Barrier & Asia Teb Barrier \\
& $\mathrm{N}(\%)$ & $\mathrm{N}(\%)$ \\
\hline Gram positive bacilli & $3(7.5)$ & $7(17.5)$ \\
Gram negative bacilli & $3(7.5)$ & $2(5.0)$ \\
Gram positive diplococcic & $1(2.5)$ & $1(2.5)$ \\
Gram positive cocci & $0(0.0)$ & $1(2.5)$ \\
Gram negative coccobacilli & $0(0.0)$ & $2(5.0)$ \\
\multicolumn{1}{c}{ Total } & $7(17.5)$ & $13(32.5)$ \\
\hline
\end{tabular}




\section{Discussion}

Creating a clean and germ-free work environment is imperative to prevent infection transmission, and ensure the safety of patients and the staff in medical and dental clinics. The cross-contamination may occur during radiography and film processing [21]. In order to break the infection transmission cycle in radiology clinics, several infection control protocols have been introduced, and their effectiveness has been evaluated [18,22,18]. The Center for Disease Control and the American Dental Association (ADA) have set some standards for infection control in radiology centers [23,24]. In this study, Asia Teb and Soredex barrier envelopes were compared, and the results showed that the rate of contamination was $32.5 \%$ in Asia Teb and $17.5 \%$ in Soredex barrier envelopes.

Currently, digital sensors have been increasingly used for intraoral radiography, but some concerns exist regarding cross-contamination [25,26]. A commonly practiced protocol for infection control in digital radiography is to use disposable barrier envelopes for PSP sensors to prevent their contamination. PSP plates protected with barrier envelopes are expected to be sterile and free from contamination. The presence of bacteria on PSP plates is unacceptable. Several previous studies have assessed the contamination of digital receptors [7,8,10,14-16]. Also, previous authors emphasized on the significance of plastic PSP plate barrier envelopes and stated that after exposure, these barrier envelopes are at high risk of contamination during radiography. Thus, certain protocols must be followed when using them [20]. In another study, it was emphasized on the risk of cross-contamination in patients associated with the use of PSP plates [16].

The current research was undertaken to compare the effectiveness of two protective barrier envelopes for PSP plates, and determine to what extent these barrier envelopes can prevent transmission of microorganisms from the oral environment to sensors. The results showed that of 80 samples, bacterial growth was noted in $17.5 \%$ of plates with Soredex, and 32.5\% of plates with Asia Teb barrier envelopes. Although this difference was not statistically significant, the bacterial growth rate was higher on plates with Asia Teb barrier envelope compared to Soredex.

In a previous study, of 44 PSP plates examined only three cases (7\%) showed bacterial contamination, and the authors concluded that contamination often occurs due to errors when removing the PSP plate from the protective barrier envelope [9]. Another research evaluated 52 PSP plates, and reported bacterial contamination in three cases. PSP plates with protective barrier envelopes should be considered as a source of infection in radiology clinics [15].

It should be noted that none of the previous studies on this topic used an effective measure to completely eliminate possible previous contamination of PSP plates [9,15]. Different reports regarding the percentage of contamination of PSP plates protected with plastic barrier envelopes indicated that the protective barrier envelopes produced by different manufacturers are different in terms of thickness and the quality of seal to prevent contamination of PSP plates. Moreover, the expertise and skills of the operator in using these sensors, changing the protective barrier envelope and removing the sensor from the envelope with sterile gloves by the operators following each time of use have been important measures for infection control. Thus, in this study, after placing the sensor into the envelope, the absence of perforation was ensured using air spray. Also, an optimal seal was ensured by the absence of any wrinkle at the edges of the barrier envelope.

On the other hand, the bisecting angle technique was used in this study for positioning of a sensor in the oral cavity, which contrasted with the previous study [15], in which a film holder was used to place a sensor. No microbiological contamination in 44 out of 52 PSP sensors was observed [15]; whereas, in this study, bacterial growth was noted in $17.5 \%$ of Soredex and $32.5 \%$ of Asia Teb samples. In the parallel 
technique, contact of film/sensor and the oral mucosa was minimized; this might explain the difference in the reported values in this study and in previous research [15].

The level of contamination of plates significantly increased if a protective barrier envelope was not used. Some authors in 2016 evaluated several types of protective barrier envelopes used in a few radiology clinics, and reported that 50 to $75 \%$ of all PSP plates without protective barrier envelopes were contaminated with bacteria when tested prior to use [27].

Each part of the oral cavity has its own unique microbial biofilm. Dominant bacteria in the saliva include Streptococcus mitis, Streptococcus sanguinis and Streptococcus salivarius, which are all Gram-positive cocci and are considered as the normal flora of the mouth [28]. In this study, the type of bacteria contaminating the PSP plates was identified by Gram staining and in both groups, Gram-positive bacilli had the highest frequency. Moreover, Gram-negative bacilli, Gram-positive and Gram-negative cocci and Gramnegative coccobacilli were also isolated from the contaminated plates. In a previous study Gram-positive bacilli and cocci were the most commonly isolated bacteria [8]. Other authors reported that staphylococci were the most commonly isolated bacteria [27].

To prevent bacterial contamination during radiography, the ADA and the Center for Disease Control have recommended heat sterilization of tools that contact the oral mucosa ideally between patients. Instruments that cannot be autoclave-sterilized or disinfected by strong disinfectants should at least be covered with protective barrier envelopes to minimize their contamination. According to the ADA, the PSP plates need to be cleaned and disinfected with an intermediate level disinfectant after each time of removal from the plastic barrier envelope. The use of ethylene oxide for sterilization of PSP plates was reported [14]. It has been recommended to rinse the mouth with chlorhexidine prior to radiography to minimize bacterial contamination [29]. A previous research showed that $82 \%$ ethanol was effective for the disinfection of plates and the elimination of bacteria and fungi [20]. Another recommendation is to use an additional latex barrier envelope over the conventional plastic envelope to minimize the risk of perforation of barrier envelope and bacterial contamination of the plates [7].

The use of two plastic barrier envelopes for each PSP plate can effectively decrease the risk of bacterial contamination of plate [27,30]. This study showed that the rate of contamination of plates was $32.5 \%$ and 17.5\% using Asia Teb and Soredex barrier envelopes; respectively. Thus, after each time of using PSP plates, they need to be disinfected using the solution recommended by the manufacturer. Future studies are recommended to assess the effect of different radiographic techniques namely the bisecting angle and the parallel technique on the level of contamination of PSP plates.

\section{Conclusion}

The contamination of photostimulable phosphor plates may still occur despite the use of plastic barrier envelopes irrespective of their type. Thus, photostimulable phosphor plates must be disinfected between patients after the removal from the protective barrier envelope, and before placing in another barrier envelope. Moreover, removal and placement of plates in protective barrier envelopes must be done with sterile disposable gloves. Also, the protective barrier envelopes should be checked prior to use to ensure the adequate sealing ability.

\section{Authors' Contributions}




\begin{tabular}{|c|c|c|}
\hline $\mathrm{NN}$ & (D) $0000-0002-2477-1761$ & Conceptualization and Methodology. \\
\hline AA & (iD) $0000-0001-5651-7824$ & Validation, Formal Analysis and Investigation. \\
\hline AK & (D) $0000-0001-5505-7571$ & Writing - Original Draft Preparation and Writing - Review and Editing. \\
\hline AK & (D) $0000-0002-6779-9756$ & Conceptualization and Writing - Review and Editing. \\
\hline SE & (1) $0000-0002-4597-121 \mathrm{X}$ & Formal Analysis and Writing - Review and Editing. \\
\hline
\end{tabular}

\section{Financial Support}

This study was supported by the Kermanshah University of Medical Sciences.

\section{Conflict of Interest}

The authors declare no conflicts of interest.

\section{Acknowledgment}

This study was derived from a thesis for a doctorate degree in Dentistry by Setareh Emadi. The authors would like to thank the personnel of Microbiology Laboratory of Kermanshah University of Medical Sciences.

\section{References}

[1] Kidd E, Pitts N. A reappraisal of the value of the bitewing radiograph in the diagnosis of posterior approximal caries. Br Dent J 1990; 169(7):195-200. https://doi.org/10.1038/sj.bdj.4807325

[2] Parks ET, Williamson GF. Digital radiography: an overview. J Contemp Dent Pract 2002; 3(4):23-39.

[3] Van Der Stelt PF. Filmless imaging: the uses of digital radiography in dental practice. J Am Dent Assoc 2005; 136(10):1379-87. https://doi.org/10.142 19/jada.archive.2005.005 1

[4] De Melo DP, Cruz AD, Melo SLS, De Farias JFG, Haiter-Neto F, De Almeida SM. Effect of different tube potential settings on caries detection using psp plate and conventional film. J Clin Diagn Res 2015; 9(4):ZC58-61. https://doi.org/10.7860/JCDR/2015/12225.5845

[5] Molander B, Gröndahl HG, Ekestubbe A. Quality of film-based and digital panoramicradiography. Dentomaxillofac Radiol 2004; 33(1):32-6. https://doi.org/10.1259/dmfr/17777906

[6] Visser H, Rödig T, Hermann KP. Dose reduction by direct-digital cephalometric radiography. Angle Orthod 2001; $71(3): 159-63$.

[7] Hokett SD, Honey JR, Ruiz F, Baisden MK, Hoen MM. Assessing the effectiveness of direct digital radiography barrier sheaths and finger cots. J Am Dent Assoc 2000; 131(4):463-7. https://doi.org/10.14219/jada.archive.2000.0202

[8] Kalathingal SM, Moore S, Kwon S, Schuster GS, Shrout MK, Plummer K. An evaluation of microbiologic contamination on phosphor plates in a dental school. Oral Surg Oral Med Oral Pathol Oral Radiol Endod 2009; 107(2):279-82. https://doi.org/10.1016/j.tripleo.2008.05.025

[9] Kuperstein AS. Defective plastic infection-control barriers and faulty technique may cause PSP plate contamination used in digital intraoral radiography. J Evid Based Dent Pract 2012; 12(2):121-2. https://doi.org/10.1016/j.jebdp.2012.03.029

[10] Negron W, Mauriello SM, Peterson CA, Arnold R. Cross-contamination of the PSP sensor in a preclinical setting. J Dent Hyg 2005; 79(3):8.

[11] Ozsevik S, Cicek E, Bodrumlu E, Guney A. Bacterial survival in the radiographic processes. Minerva Stomatol 2012; 61(4):135-40.

[12] Pinheiro S, Martoni S, Ogera R. Assessment of microbiological contamination of radiographic equipment and materials during intraoral imaging procedures. Minerva Stomatol 2012; 61(5):197-203.

[13] Bartoloni JA, Chariton DG, Flint DJ. Infection control practices in dental radiology. Gen Dent 2003; $51(3): 264-71$.

[14] Kalathingal S, Youngpeter A, Minton J, Shrout M, Dickinson D, Plummer K, et al. An evaluation of microbiologic contamination on a phosphor plate system: is weekly gas sterilization enough? Oral Surg Oral Med Oral Pathol Oral Radiol Endod 2010; 109(3):457-62. https://doi.org/10.1016/j.tripleo.2009.09.035

[15] MacDonald DS, Waterfield JD. Infection control in digital intraoral radiography: evaluation of microbiological contamination of photostimulable phosphor plates in barrier envelopes. J Can Dent Assoc 2011; 77:b93.

[16] Wenzel A, Frandsen E, Hintze H. Patient discomfort and cross-infection control in bitewing examination with a storage phosphor plate and a CCD-based sensor. J Dent 1999; 27(3):243-6.

https://doi.org/10.1016/s0300-5712(98)00063-3 
[17] American Academy of Oral and Maxillofacial Radiology infection control guidelines for dental radiographic procedures. Oral Surg Oral Med Oral Pathol 1992;73 (2):248-9. https://doi.org/10.1016/0030-4220(92)90202-2

[18] Katz JO, Cottone JA, Hardman PK, Taylor TS. Infection control protocol for dental radiology. Gen Dent 1990; 38(4):261-4.

[19] Wolfgang L. Analysis of a new barrier infection control system for dental radiographic film. Compendium 1992; 13(1):68-71.

[20] Wenzel A, Kornum F, Knudsen M, Lau EF. Antimicrobial efficiency of ethanol and 2-propanol alcohols used on contaminated storage phosphor plates and impact on durability of the plate. Dentomaxillofac Radiol 2013; 42(6):20120353. https://doi.org/10.1259/dmfr.20120353

[21] Stanczyk DA, Paunovich ED, Broome JC, Fatone MA. Microbiologic contamination during dental radiographic film processing. Oral Surg Oral Med Oral Pathol 1993; 76(1):112-9. https://doi.org/10.1016/0030-4220(93)90305-n

[22] Geist JR, Stefanac SJ, Gander DL. Infection control procedures in intraoral radiology: a survey of Michigan dental offices. Clin Prev Dent 1990; 12(2):4-8.

[23] American Dental Association Council on Scientific Affairs. The use of dental radiographs: update and recommendations. J Am Dent Assoc 2006; 137(9):1304-12. https://doi.org/10.142 19/jada.archive.2006.0393

[24] Centers for Disease Control and Prevention. 2003 CDC infection control recommendations for dental health-care settings. Compend Contin Educ Dent 2004; 25(1 Suppl):43-8, 50-3.

[25] Brian JN, Williamson GF. Digital radiography in dentistry: a survey of Indiana dentists. Dentomaxillofac Radiol 2007; 36(1):18-23. https://doi.org/10.1259/dmfr/18567861

[26] Christensen GJ. Is the current generation of technology facilitating better dentistry? J Am Dent Assoc 2011; 142(8):959-63. https://doi.org/10.14219/jada.archive.2011.0304

[27] de Souza TM, de Castro RD, de Vasconcelos LC, Pontual AD, de Moraes Ramos Perez FM, Pontual ML. Microbiological contamination inintraoral phosphor storage plates: the dilemma. Clin Oral Investig 2017; 21(1):3017. https://doi.org/10.1007/s00784-016-1790-7

[28] Heymann HO, Swift Jr EJ, Ritter AV. Sturdevant's Art and Science of Operative Dentistry. $6^{\text {th }}$. ed. London: Elsevier Health Sciences; 2012.

[29] Hunter A, Kalathingal S, Shrout M, Plummer K, Looney S. The effectiveness of a pre-procedural mouthrinse in reducing bacteria on radiographic phosphor plates. Imaging Sci Dent 2014; 44(2):149-54. https://doi.org/10.5624/isd.2014.44.2.149

[30] Thomas LP, Abramovitch K. Infection control for dental radiographic procedures. Tex Dent J 2005; 122(2):184-8. 